\title{
The Absolute Cesàro Summability of the Successively Derived Allied Series of a Fourier Series
}

\author{
·By R. Mohanty \\ (Received 12th March 1948. Read 7th May 1948.)
}

\section{§1. Introduction.}

We suppose that $f(t)$ is integrable in the Lebesgue sense $s+-\pi, \pi)$, and is periodic with period $2 \pi$. We denote its Fourier series by

$$
\frac{1}{2} a_{0}+\sum_{n=1}^{\infty}\left(a_{n} \cos n t+b_{n} \sin n t\right)=\sum_{n=0}^{\infty} A_{n}(t)
$$

Then the allied series is

$$
\sum_{n=1}^{\infty}\left(b_{n} \cos n t-a_{n} \sin n t\right)=\sum_{n=1}^{\infty} B_{n}(t) .
$$

We write

$$
\phi(t)=\frac{1}{2}\{f(x+t)+f(x-t)\}, \quad \psi(t)=\frac{1}{2}\{f(\dot{x}+t)-f(x-t)\},
$$

so that

where

$$
\begin{aligned}
& \phi(t) \sim \Sigma A_{n} \cos n t, \\
& A_{n}=A_{n}(x), \\
& \psi(t) \sim \Sigma B_{n} \sin n t, \\
& B_{n}=B_{n}(x) .
\end{aligned}
$$$$
\text { and }
$$

where $t=x$ is

$$
\Sigma\left(\frac{d}{d x}\right)^{r} B_{n}(x) \begin{cases}=\Sigma(-1)^{1 r} n^{r} B_{n} & (r \text { even }) \\ =\Sigma(-1)^{\frac{1}{2}(r+1)} n^{r} A_{n} & (r \text { odd }) .\end{cases}
$$

In a recent paper ${ }^{1}$ Bosanquet has proved the following theorem.

Theorem A. If $f(t) \in L$ in $(-\pi, \pi), a$ necessary and sufficient condition for the series (1.6) to be summable $(C, a+r)$ to sum 8 , where $a \geqq 0$ and $r$ is a positive integer, is that constants $a_{v}$ should exist such

1 Referred to as $D_{r} \bar{F} S$ in the list of references. 
that (i) the odd function $g(t)$ is integrable $(C L)$ in $(0, \pi)$ and its allied series summable $(C, a)$ at $t=0$, (ii) $g(t) / t$ is integrable $(C L ;$ in $(0, \pi)$ and 1

$$
\frac{2}{\pi} \int_{0}^{\infty} \frac{g(t)}{t} d t=s
$$

where, for $0<t<\pi$,

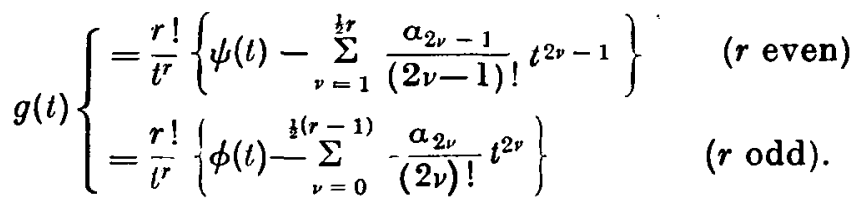

Analogous results concerning the $(C)$ summability of the $r$-th derived Fourier series and the $|C|$ summability of the first derived Fourier series have also been given by Bosanquet in $D_{r} F S$ and | $D F S \mid$ respectively. The object of the present paper is to obtain the $|C|$ analogue of Theorem $A$.

In Theorem 1 we give a general result concerning the summability $|C, a+r|$ of the $r$-th derived allied series, where $a>1$. In Theorem $2 f(l)$ is restricted to be a function of bounded variation, and a result is obtained for $a>0$.

\section{§ 2. Notation.}

We write

$$
S_{n}^{a}=\sum_{\mu=0}^{n} A_{n-\mu}^{a} u_{\mu}, \quad s_{n}^{a}=S_{n}^{a} / A_{n}^{a} \quad(\alpha>-1)
$$

for the $n$-th Cesàro partial sum and mean of order $a$ of a series $\Sigma u_{n}$, where

$$
A_{n}^{a}=\left(\begin{array}{c}
n+a \\
n
\end{array}\right) \text {. }
$$

The series $\Sigma u_{n}$ is said to be summable $(C, a)$ to $s$ if $s_{n}^{a} \rightarrow 8$, and to be summable $|C, a|$ to $s$ if, in addition, $\Sigma|\Delta s \underset{n}{a}|<\infty$.

We write $k^{\sigma}(n, t)+i \bar{k}^{\sigma}(n, t)$ for the $n$-th Cesàro mean of order $\sigma$ of the series $\frac{1}{2}+\Sigma e^{i n t}$, and require the inequalities ${ }^{2}$

$$
\left|\left(\frac{\partial}{\partial t}\right)^{k} k^{\sigma}(n, t)\right|\left\{\begin{array}{l}
\leqq A n^{k+1} \\
\biguplus A n^{k-\sigma_{t}-\sigma-1}+A n^{-1} t^{-k-2}
\end{array}\right.
$$

1 The integral in (1.7) is convergent, and taken in the $(C L)$ sense at the origin.

2 Cf. $D_{r} \bar{F} S, 64,\left|F S_{a}\right|, 519$, Obrechkoff and /ygmund. 
and

$$
\left|\left(\frac{\partial}{\partial t}\right)^{k} \bar{k}^{\sigma}(n, t)\right|\left\{\begin{array}{l}
\leqq A n^{\dot{k}+1} \\
\leqq A n^{k-\sigma} t^{-\sigma-1}+A t^{-k-1}
\end{array}\right.
$$

We write

$$
\bar{\gamma}_{\sigma}(t)=\int_{0}^{1}(1-u)^{\sigma-1} \sin t u d u \quad(\sigma>0),
$$

and require the inequalities ${ }^{1}$

$$
\left|\Delta^{k} \bar{\gamma}_{\sigma}^{(\lambda)}(t)\right|\left\{\begin{array}{l}
\leqq A t^{k} \\
\mid \leqq A n^{-\sigma} t^{k-\sigma}+A n^{-k-\lambda-1} t^{-\lambda-1} .
\end{array}\right.
$$

Here and elsewhere $\Delta u_{n}=u_{n}-u_{n+1}$, and $A$ denotes a positive number, independent of the variables but not necessarily the same at each occurrence.

The Cesàro-Lebesgue integral. Suppose that $g(t) \epsilon L$ in $(\epsilon, a)$ for every $0<\epsilon<a$ ( $a$ fixed). If

$$
\lim _{\epsilon \rightarrow 0} \int_{\varepsilon}^{a} g(t) d t
$$

exists, $g(t)$ is said to be integrable $C_{0} L$ in $(0, a)$, with the limit (2.6) as the value of the integral. If $\lambda$ is a positive integer and

(i) $G(t)=\int_{t}^{a} g(u) d u$ is integrable $C_{\lambda-1} L$ in $(0, a)$,

$$
\text { (ii) } \lim _{\epsilon \rightarrow 0} \frac{\lambda}{\epsilon^{\lambda}} \int_{0}^{\epsilon}(\epsilon-u)^{\lambda-1} G(u) d u
$$

exists, then $g(t)$ is said to be integrable $C_{\lambda} L$ in $(0, a)$, with the limit $(2: 7)$ as the value of the integral.

We write

$$
\begin{aligned}
& G_{\sigma}(t)=\frac{1}{\Gamma(\sigma)} \int_{0}^{t}(t-u)^{\sigma-1} g(u) d u \quad(\sigma>0) \\
& G_{0}(t)=g(t),
\end{aligned}
$$

and similar notation is employed with $\xi, \chi, \ldots, \Xi_{\lambda}, X_{\lambda}, \ldots$ in place of $g, G_{\lambda}$.

The absolute Cesàro-Lebesgue integral. A function $g(t)$ is said to be integrable $\left|C_{\lambda} L\right|$ in $(0, a)$, where $\lambda$ is a non-negative integer, if (i)

1 Cf. $D_{r} \bar{F} S, 64$, and $D F S, 273$. 
it is integrable $C_{\lambda} L$ in $(0, a)$, (ii) $t^{-\lambda} G_{\lambda+1}(t)$ is of bounded variation in the interval $0<t \leqq a$. The common value of the $C_{\lambda} L$ and $\left|C_{\lambda} L\right|$ integrals is then $\lim \lambda ! t^{-\lambda} G_{\lambda+1}(t)$.

If $t \rightarrow+0$

$$
\text { If } \quad t^{-\lambda-p} G_{\lambda}(t) \rightarrow \frac{\Gamma(p+1)}{\Gamma(\lambda+p+1)} s
$$

as $t \rightarrow+0$ we write $g(t) \sim s t^{p}(C, \lambda)$. If, in addition, $t^{-\lambda-p} G_{\lambda}(t)$ is of bounded variation in an interval $0<t \leqq a$ we write $g(t) \sim s t^{p}|C, \lambda|$.

Properties of the $(C L)$ and $|C L|$ integrals will be found in $D F S$, $D_{r} F S, C L$, and $|D F S|$.

\section{§ 3. Preliminary lemmas.}

In Lemmas 1 and 2 we shall suppose that $g(t) \epsilon L$ in every interval $(\delta, \Delta)$, for $0<\delta<\Delta<\infty$, and write, for $t>0$,

$$
\xi(t)=t^{\sigma-1} \int_{t}^{\infty} \frac{g(u)}{u^{\sigma}} d u \quad(\sigma>0)
$$

the integral being assumed to be convergent. It is known ${ }^{1}$ that if $g(t) \epsilon C_{\lambda+1} L$ in $(0, a)$, then

(i) $\xi(t) \epsilon C_{\lambda} L$ in $(0, a)$,

(ii) for $a \geqq 0 ; t>0$,

$$
\frac{\Xi_{a}(t)}{t^{a}}=t^{\sigma-1} \int_{t}^{\infty} \frac{G_{a}(u)}{u^{a}+\sigma} d u \text {. }
$$

Lemma 1. If $g(t) \epsilon\left|C_{\lambda+1} L\right|$ in. $(0, a)$, where $\lambda$ is a non-negative integer, then $\xi(t) \in\left|C_{\dot{\lambda}} L\right|$ in $(0, a)$.

We first prove that $\xi(t) \epsilon\left|C_{\lambda+1} L\right|$ in $(0, a)$. 'Since $g(t) \epsilon C_{\lambda+1} L$, it follows, by (i), that $\xi(t) \epsilon C_{\lambda} L$, and hence $\xi(t)_{\epsilon} C_{\lambda+1} L$. Thus in order to prove that $\xi(t) \epsilon\left|C_{\lambda+1} L\right|$ we must prove that $t^{-\lambda-1} \Xi_{\lambda+2}(t)$ is of bounded variation in the interval $0<t \leqq a$.

We have, by (3.2), ${ }^{2}$

$1 C L$, Theorems 20 and 21.

2 Defining $t-\lambda-1 \Xi \lambda+2(t)$ and $t-1 \lambda-1 G_{\lambda}+2(t)$ as zero at $t=0$. Since $g(t)$ and $\xi(t)$ are integrable $C_{\lambda+1} L$ these functions are then continuous at the origin. 


$$
\begin{aligned}
& \int_{0}^{a}\left|d\left\{t-\lambda-1 \Xi_{\lambda+2}(t)\right\}\right|=\int_{0}^{a}\left|d\left\{t^{\sigma} \int_{t}^{\infty} \frac{G_{\lambda+2}(u)}{u^{\lambda+2+\sigma}} d u\right\}\right| \\
& \leqq \int_{0}^{a}\left|d\left\{t^{\sigma} \int_{t}^{a} \frac{u^{-\lambda-1} G_{\lambda+2}(u)}{u^{1+\sigma}} d u\right\}\right|+A \\
& =\int_{0}^{a} \mid d\left\{t^{\sigma}\left[-\frac{1}{\sigma u^{\sigma}} u^{-\lambda-1} G_{\lambda+2}(u)\right]_{t}^{a}\right. \\
& \left.+\frac{t^{\sigma}}{\sigma} \int_{t}^{a} \frac{1}{u^{\sigma}} d\left(u^{-\lambda-1} G_{\lambda+2}(u)\right)\right\} \mid+A \\
& \leqq \frac{1}{\sigma} \int_{0}^{a}\left|d\left(t^{-\lambda-1} G_{\lambda+2}(t)\right)\right| \\
& +\frac{1}{\sigma} \int_{0}^{a}\left|d\left\{t^{\sigma} \int_{t}^{a} \frac{1}{u^{\sigma}} d\left(u-\lambda-1 G_{\lambda+2}(u)\right)\right\}\right|+A \\
& \leqq \frac{2}{\sigma} \int_{0}^{a}\left|d\left(t^{-\lambda-1} G_{\lambda+2}(t)\right)\right|+\int_{0}^{a} t^{\sigma-1} d t \int_{t}^{a} \frac{1}{u^{\sigma}}\left|d\left(u^{-\lambda-1} G_{\lambda+2}(u)\right)\right| \\
& +A \\
& =\frac{2}{\sigma} \int_{0}^{a}\left|d\left(t^{-\lambda-1} G_{\lambda+2}(t)\right)\right|+\int_{0}^{a} \frac{1}{u^{\sigma}}\left|d\left(u^{-\lambda-1} G_{\lambda+2}(u)\right)\right| \int_{0}^{u} t^{\sigma-1} d t \\
& +A \\
& =\frac{3}{\sigma} \int_{0}^{a}\left|d\left(t^{-\lambda-1} G_{\lambda+2}(t)\right)\right|+A \\
& <\infty \text {, }
\end{aligned}
$$

since $g(t) \epsilon\left|C_{\lambda+1} L\right|$.

We next prove that $t \xi(t)=o(1)|C, \lambda+1|$ as $t \rightarrow+0$. Integrating by parts $\lambda+2$ times, we have, as in the proof of Theorem 20 of $C L$, $i \xi(t)=-\sum_{\rho=1}^{\lambda+2} \frac{\Gamma(\sigma+\rho-1)}{\Gamma(\sigma)} \frac{G_{\rho}(t)}{t^{\rho-1}}+\frac{\Gamma(\sigma+\lambda+2)}{\Gamma(\sigma)} t^{\sigma} \int_{t}^{\infty} \frac{G_{\lambda+2}(u)}{u^{\lambda}+\zeta} d u$.

Since $g(t) \epsilon\left|C_{\lambda+1} L\right|$ in $(0, a)$, it follows ${ }^{2}$ that $G_{\rho}(t)=o\left(t^{\rho-1}\right)|C, \lambda+2-\rho|$, for $\rho=1,2, \ldots, \lambda+2$, and hence ${ }^{2}$ that $G_{\rho}(l) / t^{\rho-1}=o(1)|C, \lambda+2-\rho|$. Also the integral in (3.3) is $o(1)$ as $t \rightarrow+0$, as in the proof of Theorem 20 of $C L$, and we have just proved that it is of bounded variation in $0<t \leqq a$. It follows that all the terms in (3.3) are o(1) $|C, \lambda+1|$, i.e. that $t \xi(t)=o(1)|C, \lambda+1|$.

Finally we deduce that $\xi(t) \epsilon C_{\lambda} L$. Writing $\chi(t)=t \xi(t)$, we have ${ }^{3}$

${ }^{3}|D F S|, 18$.

${ }^{2} \mid$ DFS $\mid$, Lemma 1.

$3 \mathrm{CL}, \mathbf{5 5}$. 


$$
\mathrm{X}_{\lambda+1}(t)=t \Xi_{\lambda+1}(t)-(\lambda+1) \Xi_{\lambda+2}(t)
$$

Hence

$$
t^{-\lambda} \Xi_{\lambda+1}(t)=t^{-\lambda-1} X_{\lambda+1}(t)+(\lambda+1) t^{-\lambda-1} \Xi_{\lambda+2}(t) .
$$

But we have just proved that the two expressions on the right of (3.5) are of bounded variation in $0<t \leqq a$, and hence so is $t^{-\lambda} \Xi_{\lambda+1}(t)$. We have also observed that $\xi(t) \epsilon C_{\lambda} L$, and thus it follows that $\xi(t) \epsilon\left|C_{\lambda} L\right|$.

LEMMA 2. If $g(t) \epsilon|C L|$ in $(0, a), \lambda$ is a non-negative integer and $\sigma>0$, then a necessary and sufficient condition that $g(t) / t^{\sigma} \epsilon\left|C_{\lambda} L\right|$ in $(0, a)$ and

$$
\int_{0}^{\infty} \frac{g(u)}{u^{\sigma}} d u=l
$$

is that $G_{\lambda}(t) / t^{\lambda}+\sigma$ should be integrable $L$ in $(0, a)$, and

$$
\int_{0}^{\infty} \frac{G_{\lambda}(u)}{u^{\lambda+\sigma}} d u=\frac{\Gamma(\sigma)}{\Gamma(\lambda+\sigma)} l
$$

Necessity. Suppose that $g(t) / l^{\sigma_{\epsilon}}\left|C_{\lambda} L\right|$ and that (3.6) holds, i.e. that $\xi(t) / t^{\sigma-1} \rightarrow l|C, \lambda|$ as $t \rightarrow+0$. It follows ${ }^{1}$ that $\xi(t) \sim l t^{\sigma-1}|C, \lambda|$ as $t \rightarrow+0$, i.e. that

$$
\frac{\Xi_{\lambda}(t)}{t^{\lambda+\sigma-1}} \sim \frac{\Gamma(\sigma)}{\Gamma(\lambda+\sigma)} l(C, 0)
$$

which, by (3.2), is (3.7).

Sufficiency. Since $\xi(t) \epsilon|C L|$ in $(0, a)$, by Lemma 1 , the sufficiency is established by reversing the argument.

Lemma 3. If $k$ is a non-negative integer, and $\Sigma u_{n}$ is summable $|C, k|$ to $s$, then

$$
\frac{2 \sigma}{\pi} \int_{0}^{\infty} \frac{1}{u} \lim _{\rho} \Sigma u_{n} \bar{\gamma}_{\sigma}(n u) \rho^{n} d u=s
$$

for $\sigma>k$, where the integral in (3.9) is an absolutely convergent Lebesgue integral.

We have".

1 DFS $\mid$, Lemma 1 .

2 Cf. $D_{r} F S$, Lemma 4. We write $s_{-1}^{k}=0$. 


$$
\begin{aligned}
S(t) & =\lim _{\rho \rightarrow 1-0} \sum u_{n} \bar{\gamma}_{\sigma}(n t) \rho^{n} \\
& =\Sigma S_{n}^{k} \Delta^{k+1} \bar{\gamma}_{\sigma}(n t) \\
& =\sum s_{n}^{k} A_{n}^{k} \Delta^{k+1} \bar{\gamma}_{\sigma}(n t) \\
& =-\sum \Delta s_{n-1}^{k} J(n, t), \\
J(n, t) & =\sum_{\nu=n}^{\infty} A_{\nu}^{k} \Delta^{k+1} \bar{\gamma}_{\sigma}(\nu t) .
\end{aligned}
$$

where

By the analogue for ordinary Cesàro summability, (3.9) certainly holds with the integral interpreted as a $C_{0} L$ integral in $(0, a)$. It will therefore be sufficient to prove that

$$
\int_{0}^{\infty} t^{-1}|S(t)| d t<\infty
$$

We have

$$
\begin{aligned}
\int_{0}^{\infty} t^{-1}|S(t)| d t & \leqq \int_{0}^{\infty} t^{-1} \Sigma\left|\Delta s_{n-1}^{k} J(n, t)\right| d t \\
& =\Sigma\left|\Delta_{s_{n-1}^{k}}\right| \int_{0}^{\infty} t^{-1}|J(n, t)| d t .
\end{aligned}
$$

Now

$$
|J(n, t)|\left\{\begin{array}{l}
\leqq A n^{k+1} t^{k+1} \\
\leqq A n^{-1} t^{-1}+A n^{k-\sigma} t^{k-\sigma} .
\end{array}\right.
$$

For

$$
J(0, t)=\sum_{\nu=0}^{\infty} A_{\nu}^{k} \Delta^{k+1} \bar{\gamma}_{\sigma}(\nu t)=\sum_{\nu=0}^{\infty} \Delta \bar{\gamma}(\nu t)=0,
$$

and hence

$$
\begin{aligned}
J(n, t) & =J(n, t)-J(0, t)=-\sum_{\nu=0}^{n-1} A_{\nu}^{k} \Delta^{k+1} \bar{\gamma}_{\sigma}(\nu t) \\
& \cdot \sum_{\nu=1}^{n} O\left(\nu^{k}\right) O\left(t^{k+1}\right)=O\left(n^{k+1} t^{k+1}\right) .
\end{aligned}
$$

On the other hand, we have

$$
\begin{aligned}
\sum_{\nu=n}^{\infty} A_{\nu}^{k} \Delta^{k+1} \bar{\gamma}_{\sigma}(\nu t) & =A_{n}^{k} \Delta^{k} \bar{\gamma}_{\sigma}(n t)+\sum_{\nu=n+1}^{\infty} A_{\nu}^{k-1} \Delta^{k} \bar{\gamma}_{\sigma}(\nu t) \\
& =O\left(n^{k}\right)\left\{O\left(n^{-k-1} t^{-1}\right)+O\left(n-\sigma t^{k-\sigma}\right)\right\} \\
& +\sum_{\nu}^{\infty} O\left(\nu^{k-1}\right)\left\{O\left(\nu^{-k-1} t^{-1}\right)+O\left(\nu-\sigma t^{k-\sigma}\right)\right\} \\
& =O\left(n^{-1} t^{-1}\right)+O\left(n^{k-\sigma} t^{k-\sigma}\right)
\end{aligned}
$$

for $\sigma>k$. 
It follows from (3.12) that, for $n \geqq 0$,

$$
\begin{aligned}
& \int_{0}^{\infty} t^{-1}|J(n, t)| d t=\int_{0}^{n-1} O\left(n^{k+1} t^{k}\right) d t+\int_{n-1}^{\infty}\left\{O\left(n^{-1} t^{-2}\right)\right. \\
& \left.\quad+O\left(n^{k-\sigma} t^{k-\sigma-1}\right)\right\} d t \\
& \text { for } \sigma>k .
\end{aligned}
$$

Thus $\quad \int_{0}^{\infty} t^{-1}|S(t)| d t \leqq A \Sigma\left|\Delta s_{n-1}^{k}\right|<\infty$.

Lemma 4. If $g(t) \epsilon\left|C_{k} L\right|$ in $(0, \pi)$, where $k$ is a non-negative integer, and if

$$
a_{n}+i \beta_{n}=\int_{0}^{\pi} g(t) e^{i n t} d t,
$$

then $a_{n}$ and $\beta_{n}$ are o(1) $\mid C$, o $\mid$ as $n \rightarrow \infty$, for $\sigma>k+1$.

Writing $a_{n}^{\sigma}, c^{\sigma}(n, t)$ for the $n$-th Cesàro means of order $\sigma$ of the sequences $\alpha_{n}$ and $\cos n t$ respectively, we have ${ }^{1}$

$$
\begin{aligned}
& a_{n}^{\sigma}=\int_{0}^{\pi} g(t) c^{\sigma}(n, t) d t \\
& =\left[\sum_{\rho=0}^{k}(-1)^{\rho} G_{\rho+1}(t)\left(\frac{\partial}{\partial t}\right)^{\rho} c^{\sigma}(n, t)\right]_{0}^{\pi} \\
& +(-1)^{k+1} \int_{0}^{\pi} G_{k+1}(t)\left(\frac{\partial}{\partial t}\right)^{k+1} c^{\sigma}(n, t) d t \\
& =\sum_{\rho=0}^{k}(-1)^{\rho} G_{\rho+1}(\pi)\left\{\left(\frac{\partial}{\partial t}\right)^{\rho} c^{\sigma}(n, t)\right\}_{t=\pi} \\
& \quad+(-1)^{k+1} \int_{0}^{\pi} d\left\{t-k G_{k+1}(t)\right\} \int_{t}^{\pi} v^{k}\left(\frac{\partial}{\partial v}\right)^{k+1} c^{\sigma}(n, v) d v,
\end{aligned}
$$

where $t^{-k} G_{k+1}(t)$ is defined as zero-for $t=0$. Hence it follows that

$$
\begin{aligned}
\Sigma\left|\Delta a_{n}^{\sigma}\right| \leqq & \sum_{\rho=0}^{k}\left|G_{\rho+1}(\pi)\right| \Sigma\left|\Delta\left(\frac{\partial}{\partial t}\right)^{\rho} c^{\sigma}(n, t)\right| t=\pi \\
& +\int_{0}^{\pi}\left|d\left\{t^{-k} G_{k+1}(t)\right\}\right| \Sigma\left|\int_{t}^{\pi} v^{k} \Delta\left(\frac{\partial}{\partial v}\right)^{k+1} c^{\sigma}(n, v) d v\right|
\end{aligned}
$$

Cf. $D_{r} \bar{F} S$, Lemma 9 . 
Now

$\left|\Delta\left(\frac{\partial}{\partial t}\right)^{\rho} c^{\sigma}(n, t)\right|\left\{\begin{array}{l}\leqq A n^{\rho} t \\ \leqq A n^{\rho-\sigma} t^{-\sigma+1}+A n^{-3} t^{-\rho-2},\end{array}\right.$

$\left|\int_{0}^{t} v^{k} \Delta\left(\frac{\partial}{\partial v}\right)^{k+1} c^{\sigma}(n, v) d v\right| \leqq A n^{k+1} t^{k+2}$,

$\left|\int_{t}^{\pi} v^{k} \Delta\left(\frac{\partial}{\partial v}\right)^{k+1} c^{\sigma}(n, v) d v\right| \leqq A n^{k-\sigma} t^{k-\sigma+1}+A n^{-3} t^{-2}$

and

$\left|\int_{0}^{\pi} v^{k} \Delta\left(\frac{\partial}{\partial v}\right)^{k+1} c^{\sigma}(n, v) d v\right| \leqq A n^{k-\sigma}+A n^{-3}$.

The case $\rho=0$ of (3.15) is given in $|D F S|$, and the general case is obtained similarly by use of (2.2). ${ }^{1}$ We obtain (3.16) immediately from (3.15) i, while (3.17) follows from (3.15) ii after integration by parts, and (3.18) follows from (3.15) after repeated integrations by parts.

The first term on the right of (3.14) is

$$
\sum_{\rho=0}^{k} \Sigma\left\{O\left(n^{\rho-\sigma}\right)+O\left(n^{-3}\right)\right\}<\infty .
$$

Also

$$
\int_{0}^{\pi}\left|d\left\{t^{-k} G_{k+1}(t)\right\}\right|<\infty,
$$

since $g(t) \in\left|C_{k} L\right|$. It will therefore be sufficient to prove that

$$
J(t)=\Sigma\left|\int_{t}^{\pi} v^{k} \Delta\left(\frac{\partial}{\partial v}\right)^{k+1} c^{\sigma}(n, v) d v\right|
$$

is bounded in $0 \leqq t \leqq \pi$.

Disposing of the case $t=0$ by (3.18), we write, for $0<t \leqq \pi$,

$$
J(t)=\sum_{n<t^{-1}}+\sum_{n>t^{-1}}=\Sigma_{1}+\Sigma_{2}
$$

Thus, by (3.16) and (3.18),

$$
\begin{aligned}
\Sigma_{1} & =\sum_{n<t^{-1}}\left\{O\left(n^{k+1} t^{k+2}\right)+O\left(n^{k-\sigma}\right)+O\left(n^{-3}\right)\right\} \\
& =O(1)
\end{aligned}
$$

for $\sigma>k+1$, and, by (3.17),

$$
{ }^{\sigma}(n, t)=\frac{\sigma}{n+\sigma}\left\{l^{\sigma}-1(n, t)-\frac{1}{2}\right\}
$$




$$
\begin{aligned}
\Sigma_{2} & =\sum_{n>t^{-1}}\left\{O\left(n^{k-\sigma} t^{k-\sigma+1}\right)+O\left(n^{-3} t^{-2}\right)\right\} \\
& =O(1)
\end{aligned}
$$

for $\sigma>k+1$.

This completes the proof of the result for $a_{n}$; that for $\beta_{n}$ is similar, (2.3) taking the place of $(2.2)$.

\section{\$4. The main theorem.}

Theorem 1. If $f(t)$ is integrable $L$ in $(-\pi, \pi)$ and periodic with period 2 $\pi$, a necessary and sufficient condition that the series (1.6) should be summable $|C, \alpha+r|$ to the sum $s$, where $\alpha>1$ and $r$ is $a$ positive integer, is that constants $a_{\nu}$ should exist such that (i) the odd function $g(t)$ is integrable $|C L|$ in $(0, \pi)$ and its allied series summable $|C, \alpha|$ at $t=0$, (ii) $g(t) / t$ is integrable $|C L|$ in $(0, \pi)$ and (1.7) holds, ${ }^{1}$ where $g(t)$ is defined by (1.8).

The proof depends on the following lemmas.

Lemma 5. If $f(t) \epsilon L$ and the series (1.6) is summable $|C|$ to $s$, then there exist constants $\alpha_{\nu}$ such that (i) $g(t) / t \in|C L|$ in $(0, \pi)$, where $g(t)$ is given by (1.8), (ii) (1.7) is satisfied.

The proof is similar to that of Lemma 10 of $D_{r} \bar{F} S$, but with Lemmas 2 and 3 of the present paper taking the place of Lemmas 5 and 8 respectively of $D_{r} \bar{F} S$.

LEMMA 6. If $g(t)$ is odd and $g(t) / t \in|C L|$ in $(0, \pi)$, then $g(t) \epsilon|C \dot{L}|$ in $(0, \pi)$ and its allied series is summable $|C|$ at $t=0$.

The proof is similar to that of the sufficiency part of Lemma 11 of $D_{r} \bar{F} S$, but with Lemma 2 of $|D F S|$ and Lemma 4 of the present paper taking the place of Lemmas 2 and 9 respectively of $D_{r} \bar{F} S$, and with Lemma 3 of $D_{r} \bar{F} S$ replaced by its $|C|$ analogue. ${ }^{2}$

LemMa 7. If constants $a_{y}$ exist such that the odd function $g(t)$, defined by $(1.8)$ in $(0, \pi)$, is integrable $|C L|$ in $(0, \pi)$, then constants $\beta_{v}$

1 The integral in (1.7) being convergent and existing as a $|C L|$ integral at the origin.

2 The proof of this is similar to that of Lemma 2 of $|D F S|$. 
exist such that the odd function $G(t)$ is integrable $|C L|$ in $(0, \pi)$, where, for $0<t<\pi$,

$$
G(t)\left\{\begin{array}{l}
=\frac{r !}{\left(2 \sin \frac{1}{2} t\right)^{r}}\left\{\psi(t)-\sum_{v=1}^{3 r} \frac{\beta_{2 v-1}}{(2 v-1) !} \sin ^{2 v-1} t\right\} \\
=\frac{r !}{\left(2 \sin \frac{1}{2} t\right)^{r}}\left\{\phi(t)-\sum_{\nu=0}^{t(r-1)} \frac{\beta_{2 v}}{(2 \nu) !} \sin ^{2 \nu} t\right\}
\end{array}\right.
$$

and if the allied series of either $g(t)$ or $G(t)$ is summable $|C, a|$ at $t=0$, where $a>0$, then so is that of the other.

The proof is similar to that of Lemma 12 of $D_{r} \bar{F} S$, but with Lemma 2 of $|D F S|$ and the case $k=0$ of Lemma 4 of the present. paper taking the place of Lemma 2 of $D_{r} \overline{F S}$ and the RiemannLebesgue theorem respectively.

Lemma 8. If (i) $G(t) \epsilon|C L|$ in $(0, \pi)$, (ii) $t^{r} G(t) \epsilon L$ in $(0, \pi)$, where $r$ is a positive integer, and if

$$
\beta(\mu)=\frac{2}{\pi} \int_{0}^{\pi} G(t) \sin \mu t d t,
$$

then if one of the series $\Sigma \beta(n)$ and $\Sigma \beta\left(n-\frac{1}{2} r\right)$ is summable $|C, \alpha|$, where $a>1$, so is the other.

The proof is similar to that of Lemma 13 of $D_{r} \bar{F} S$, but with Lemma 2 of $|D F S|$ and the case $k=0$ of Lemma 4 of the present paper taking the place of Lemma 2 of $D_{r} \bar{F} S$ and the RiemannLebesgue theorem respectively.

Lemma 9. If $G(t)$ is defined by (4.1) and $\beta(\mu)$ by (4.2), then $n^{r} \Delta^{r} \beta\left(n-\frac{1}{2} r\right)=r !(d / d x)^{r}\left(b_{n} \cos n x-a_{n} \sin n x\right) \quad$ for $n \geqq r$.

This is Lemma 14 of $D_{r} \bar{F} S$.

Lemma 10. If $\Sigma u_{n}$ is summable $|C|$, then a necessary and sufficient condition for $\Sigma n^{r} \Delta^{r} u_{n}$ to be summable $|C, a+r|$, where $a>-1$ and $r$ is a positive integer, is that $\Sigma u_{n}$ be summable $|C, a|$.

The proof is similar to that of Lemma 14 of $D_{r} \overline{F S} .^{1}$

Proof of Theorem 1. Necessity. Suppose that the series (1.6) is summable $|C, a+r|$ to sum $s$, where $a>1$. Then, by Lemma 5 , constants $a_{\nu}$ exist such that $g(t) / t \in|C L|$ in $(0, \pi)$, where $g(t)$ is given

1 The case $r=1$ was given in $|D F S|$ (Lemma 8). See also Chow, and Bosanquet and Chow. 
by (1.8), and (1.7) holds. It follows, by Lemma 6, that $g(t) \epsilon|C L|$ in $(0, \pi)$ and its allied series is summable $|C|$ at $t=0$.

Now, by Lemma $7, g(t)$ defines a function $G(t)$, given by (4.1), which is integrable $|C L|$ in $(0, \pi)$, and its allied series is also summable $|C|$ at $t=0$, i.e. $\Sigma \beta(n)$ is summable $|C|$, where $\beta(n)$ is defined by (4-2). It follows, by Lemma 8, that $\Sigma \beta\left(n-\frac{1}{2} r\right)$ is summable $|C|$. Now, by Lemma 9, our hypothesis is that $\Sigma n^{r} \Delta^{r} \beta\left(n-\frac{1}{2} r\right)$ is summable $|C, a+r|$. Therefore, by the necessity part of Lemma $10, \Sigma \beta\left(n-\frac{1}{2} r\right)$ is summable $|C, a|$, and, by Lemma 8 , so also is $\Sigma \beta(n)$, i.e. the allied series of $G(t)$ is summable $|C, a|$ at $t=0$. It follows, by Lemma 7, that the allied series of $g(t)$ is summable $|C, a|$ at $t=0$.

Thus the conditions are necessary.

Sufficiency. Suppose that constants $a_{\nu}$ exist such that the odd function $g(t)$, given by $(1.8)$, is integrable $|C L|$ in $(0, \pi)$, that its allied series is summable $|C, a|$ at $t=0$, where $\alpha>1$, and that (1.7) holds, the function $g(t) / t$ being integrable $|C L|$ in $(0, \pi)$. Then $g(t)$ defines $G(t)$, given by (4.1), which is also integrable $|C L|$, and its allied series is summable $|C, a|$ at $t=0$, i.e. $\Sigma \beta(n)$ is summable $|C, a|$. Then by Lemma $8, \Sigma \beta\left(n-\frac{1}{2} r\right)$ is summable $|C, a|$ and, by.Lemma 9 and the sufficiency part of Lemma 10, the series (1.6) is summable $|C, a+r|$. Finally, by (1.7) and the necessity part of the theorem, the sum is $s$.

This completes the proof of the theorem.

\section{§ 5. Additional result.}

THEOREM 2. If the function $f(t)$ in Theorem 1 is of bounded variation in $(-\pi, \pi)$, then the result of Theorem 1 holds for $a>0$.

The proof of Theorem 2 is similar to that of Theorem 1 except that at the points in the proof where we used the case $k=0$ of Lemma 4 we now use the following lemma.

LeMma 11. If $g(t)$ is of bounded variation in $(0, \pi)$, and $\alpha_{n}, \beta_{n}$ are given by (3.13), then $\alpha_{n}$ and $\beta_{n}$ are $o(1)|C, \sigma|$ as $n \rightarrow \infty$, for $\sigma>\mathbf{0}$.

To prove the result for $a_{n}$ we suppose, as we may, that $g(t)$ is even and let

$$
g(t) \sim \frac{2}{\pi} \Sigma a_{n} \cos n t
$$

Since $g(t)$ is of bounded variation in $(0, \pi)$ it follows from a theorem of Bosanquet ${ }^{1}$ that $\Sigma \alpha_{n}$ is summable $|. C, \sigma|$, for $o>0$, and hence $a_{n}=o(1)|C, \sigma|$.

1 I F.S. I Theorem 1. 
To prove the result for $\beta_{n}$ we write

$$
\begin{aligned}
\beta_{n}-\beta_{n-1} & =\int_{0}^{\pi} g(t)\{\sin n t-\sin (n-1) t\} d t \\
& =\int_{0}^{\pi} g(t) \sin t \cos n t d t+\int_{0}^{\pi} g(t)(1-\cos t) \sin n t d t \\
& =\gamma_{n}+\delta_{n} .
\end{aligned}
$$

Then, by the same theorem of Bosanquet and a theorem of Bosanquet and Hyslop, ${ }^{1} \Sigma \gamma_{n}$ and $\Sigma \delta_{n}$ are both summable $|C, \sigma|$, for $\sigma>0$. Since $\beta_{n}=o(1)$, by the Riemann-Lebesgue theorem, the result follows.

Finally I should like to express my thanks to Dr L. S. Bosanquet for his suggestions and criticisms.

' Bosanquet and Byslop, Theorem, 4.

\section{REFERENCES.}

S. P. Bhatnagar, "On the Fourier coefficients of a discontinuous function," Proc. Edinburgh Math. Soc. (2), 6 (1939-41), 231-256.

L. S. Bosanquet, $\left|F S_{0}\right|$, "Note on the absolute summability $(C)$ of a Fourier series," Journal London Math. Soc., 11 (1936), 11-15.

- $\left|F S_{\alpha}\right|$, “The absolute Cesàro summability of a Fourier series," Proc. London Math. Soc. (2), 41 (1936), 517-528.

—— , DFS, "Note on differentiated Fourier series," Quart. J. of Math. (Oxford Series), 10 (1939), 67-74.

- $D_{r} F S$, "A solution of the Cesàro summability problem for successively derived Fourier series," Proc. London Math. Soc. (2), 46(1940), 270-289.

— , $|D F S|$, "The absolute Cesàro summability problem for differentiated Fourier series," Quart J. of Math. (Oxford Series), 12 (1941), 15-25.

—_ _ $C L$, "Some properties of Cesàro-Lebesgue integrals," Proc. London Math. Soc. (2), 49 (1945), 40-62.

, $D_{r} \bar{F} S$, "The Cesàro summability of the successively derived allied series of a Fourier series," Proc. London Math. Soc. (2), 49 (1945), 63-76. 
L. S. Bosanquet and H. C. Chow, "Some analogues of a theorem of Andersen,", Journal London Math. Soc., 16 (1941), 42-48.

- — and J. M. Hyslop, "On the absolute summability of the allied series of a Fourier series," Math. Zeit., 42 (1937), 489-512.

H. C. Chow, "On the absolute summability $(C)$ of power series," Journal London Math. Soe., 14 (1939), 101-112.

G. H. Hardy, "Notes on some points in the integral calculus (LXVI): The arithmetic mean of a Fourier constant," Messenger of Math., 58 (1928), 50-52.

- - and W. W. Rogosinski, "Notes on Fourier series (IV): Summability $\left(\mathrm{R}_{2}\right)$," Proc. Cumbridge Phil. Soc., 43 (1947), 10-25.

N. Obrechkoff, "Sur la sommation des séries trigonométriques de Fourier par les moyennes arithmétiques," Bull. de la Soc. Math. de France, 62 (1934), 84-109 and 167-184.

A. Zyginund, "Sur un théorème de M. Gronwall," Bull. de l'Acad. polonaise (Cracovie), A (1925), 207-217.

\section{UNIVERSTTY COLLEgE,}

LONDON. 